\title{
Program Design Considerations for Leadership Training for Dental and Dental Hygiene Students
}

\author{
Russell S. Taichman, D.M.D., D.M.Sc.; Joseph W. Parkinson, D.D.S., F.A.G.D.; \\ Bonnie A. Nelson, D.D.S.; Barbara Nordquist, R.D.S.A.; Daphne C. Ferguson-Young, \\ D.D.S., M.S.P.H.; Joseph F. Thompson Jr., M.H.A.
}

Abstract: Since leadership is an essential part of the oral health professions, oral health educators can play an essential role in establishing a culture of leadership and in mentoring students to prepare them for future leadership roles within the profession. However, leadership training for oral health professionals is a relatively new concept and is frequently not found within dental and dental hygiene curricula. The purpose of this article is to propose several models for leadership training that are specific to the oral health professions. The authors hope that providing an overview of leadership programs in academic dental institutions will encourage all U.S. and Canadian dental schools to begin developing a culture that promotes leadership development.

Dr. Taichman is Professor, Department of Periodontics and Oral Medicine, Scholars Program in Dental Leadership, University of Michigan School of Dentistry; Dr. Parkinson is Director of Quality Assurance, Department of Restorative Dentistry, University of Missouri-Kansas City School of Dentistry; Dr. Nelson is Assistant Professor and Chair of Pediatric Dentistry, Loma Linda University School of Dentistry, and Department of Hospital Dentistry, Loma Linda University Medical Center; Ms. Nordquist is Vice President, Academic and Professional Relations, KaVo Group; Dr. Ferguson-Young is Associate Professor, Department of Restorative Dentistry, and Director, GPR Program, Meharry Medical College School of Dentistry; and Mr. Thompson is Assistant Dean for Finance and Administration, Medical University of South Carolina College of Dental Medicine. Direct correspondence and requests for reprints to Dr. Russell S. Taichman, Department of Periodontics and Oral Medicine, School of Dentistry, University of Michigan, 1011 North University Ave., Ann Arbor, MI 48109-1078; 734-764-9952 phone; rtaich@umich.edu.

Keywords: dental education, allied dental education, leadership, leadership training, interprofessional education

Submitted for publication 2/4/11; accepted 5/25/11

$\mathrm{T}$ The Hippocratic Oath represents the cornerstone of ethical behavior that all health care professionals aspire to uphold. While the oath itself has been adapted for modern times and specifically for use in dentistry, the central tenet remains that the healer will act in the best interests of his or her patients. ${ }^{1}$ This implies that dental/oral health professionals will also serve as advocates for their patients' oral health. Yet in the United States and other countries, optimal oral health is often viewed as a luxury or entitlement. In addition, many influences are redefining the scope and the practice of the oral health professions, which may or may not represent our patients' best interests. Thus, a need has arisen as to who will lead the charge for our patients if not those of us in the business of dental education.

One venue in which dental educators can advocate for oral health is to inspire and provide the tools for our students to seek out and assume positions of influence and leadership. In so doing, future practitioners may find opportunities to advocate for and influence policy on behalf of their patients. Therefore, we advocate for the development and incorporation of leadership training programs within the curricula of dental and dental hygiene schools. The purpose of this article is to provide guidance on how leadership training for our students might be achieved.

\section{Leadership Training for Dental and Dental Hygiene Students}

Developing leadership skills is a multifaceted undertaking requiring the ability to understand complex problems. ${ }^{2}$ Humility and the ability to articulate a plan in alignment with a strategic vision are also competencies associated with strong leadership. ${ }^{3}$ True leadership is the ability to see the entire landscape through which a team must navigate, to listen objectively, to organize and manage projects by team members, and to monitor progress towards objectives. Competencies define what skills and 
knowledge are required for the job. Leadership as a competence can be defined as the process of managing and serving as an advocate to help others achieve particular outcomes.

It is important to draw a distinction between leadership and management. The major difference is that leadership is focused on vision and management is focused on implementation. While the ability to implement a plan is a critical aspect of leadership, management skills are a subset of leadership skills. Good managers may not be good leaders, and leaders with poor management skills may not be effective leaders.

Leadership organizations have proposed leadership competencies ranging from knowledge and skills in finance to self-reflection and emotional intelligence. Collins-Nakai cites an investigation conducted for the Canadian Medical Association (CMA) that concluded physicians' leadership development programs should be based upon the idea that "old models of leadership are irrelevant and unappealing" for its members. ${ }^{4}$ Most specifically, according to Collins-Nakai, physicians reported a need for "versatile content as well as flexible and collaborative delivery including non-classroom options." In addition, Collins-Nakai reported that members of the CMA emphasized learning principles with an appropriate balance of self-reflection, theory, practice, and skills. Clearly, as in any aspect of education, including leadership development in the context of dentistry, attainment of competence is best served in an active learning process supported by self-reflection and peer/mentor input. ${ }^{5-8}$

What exactly should be the competencies/ characteristics that future oral health leaders should strive to attain?2,3,9-13 Undoubtedly, "highly successful leaders possess unconditional integrity, charisma, and a powerful drive for authenticity." 14 This concept is not necessarily different from what is required of leaders in other professions. Moreover, DePaola and Slavkin summarized the proceedings of a conference supported by the Maternal and Child Health Bureau on "The Future of Maternal and Child Health Leadership." ${ }^{15}$ In this work, four primary cross-cutting competencies across all health professions were described. Starting from these overarching themes as a basis of discussion, we see significant latitude that can be incorporated into individual program development.

A fundamental issue that is often raised in this context is to what extent is it possible to teach or model these skills in a dental school setting. If leadership is defined by individuals who represent "high charisma individuals who create followers through personal magnetism," then clearly this will be a difficult task. ${ }^{15}$ Dental and dental hygiene students are not recruited exclusively for their leadership skills or personal magnetism. If we define the development of leadership skills as thinking and acting outside of conventional frameworks by individuals who "confront and challenge old patterns and spearhead new ones, at any level," then we can achieve this goal. ${ }^{15}$ Leadership is often defined by individuals who are willing to remove themselves from the doctor-patient relationship and examine problems at a systems level. ${ }^{4}$ There are four parts of the framework of crossdisciplinary competencies.

\section{Communication Skills}

If dental students are to serve as leaders of the oral health care professions, they must develop skills to motivate and inspire stakeholders, including future employees, to peak performance through effective communication. In clinical practice, competence in this area is likely to produce rewarding results in and of itself and have a positive impact on productivity, profitability, retention, commitment, and engagement. Networking is an extremely important element of being able to communicate. It facilitates interactions of common interest. Negotiation skills are also essential in a practice setting as they enable the leader to conduct business in a mutually beneficial environment, yet it goes beyond mastery of communication in that good negotiation skills can bring groups together and people with resources and influence to create options that may not exist otherwise.

While verbal communication skills are critical for effective leadership, the ability to communicate with writing skills is also essential. Effective writing enables a leader to convey information, concepts, and points of view in a professional and convincing manner to diverse audiences across time and location. Similarly, the ability to utilize current and emerging technologies is crucial to help a leader evaluate and disseminate information.

\section{Ability for Self-Reflection}

One of the most important characteristics of communication is the ability to share a vision. Many characteristics are required to exercise leadership, but as a leader "you must clarify your own vision of the 
future before you enlist others in a shared vision ... if you don't care deeply for and about something or someone, then how can you expect others to feel any sense of conviction?"16 The Merriam-Webster dictionary defines vision as "a thought, concept, or object formed by the imagination." Vision most effectively comes from self-reflection and is often the by-product of self-directed learning.

When it comes to leadership vision, action is also important. In Strengthening Departmental Leadership: A Team-Building Guide for Chairs in Colleges and Universities, Lucas puts it this way: "An important leadership characteristic is the ability to create a shared vision. A vision in this instance is simply a word picture of the future that individuals want to create. A vision motivates because it provides a challenge that can mobilize the organization and its people and because it increases self-esteem among the faculty. An effective vision appeals to both intellect and the emotions; the two combined can create strong positive motivations." ${ }^{17}$ Moreover, "effective leaders help articulate a vision, set standards for performance, and create focus and direction." 18

One aspect of a leadership position is the leader's ability to change the vision into action. There is a Japanese proverb that says, "Vision without action is a daydream, action without vision is a nightmare." 19 Detsky expanded an academic vision "to include several related concepts: mission, objectives, and enthusiasm." ${ }^{20}$ Leaders' vision can come from inside themselves and from many other sources: superiors, interest groups, and peers. The leader must evaluate carefully all facts that are given to him or her and have the insight to realize that his or her vision may have to change. Without enthusiasm, the leader will never obtain the support needed to move forward. A leader's visions are not always popular but can be particularly important in times of crisis and uncertainty. "An effective leader can articulate a vision with enthusiasm to make it happen, then give the credit to all who participate," Detsky has written. ${ }^{20}$ In fact, it is often said that the best leaders are the best followers.

\section{Critical Thinking and Problem- Solving Skills}

It is likely that the breakthroughs with the greatest impact for the profession will come from those leaders who take a fresh approach to emerging opportunities. Kanter suggests that to "foster innovation and transformation, leaders should focus on impact, not inputs. Leaders must identify unsolved problems, map the wider system influencing results, and determine weak links to strengthen or gaps to fill." ${ }^{21}$

The paradox that emerged throughout most of the twentieth century is that organized dentistry often sought to set itself apart from other aspects of health care. As a profession, we have often sought to be a separate and distinct entity. In part, this has been due to the notion that the profession can best regulate itself and that there was an economic advantage to this position. However, most dental schools function within a university setting in which scholarship, creativity, and critical thinking are considered the coin of the realm. Within dental schools this has often lead to a schism between clinician educators and those whose contributions focused on research scholarship. However, to develop our profession, all of our resources must be utilized to take advantage of the development of information and innovations in the present and future. Therefore, educators must value the ability to think critically. Just as institutions of higher learning provide opportunities for faculty members to practice these skills as part of their professional training, so too must we provide opportunities and reward students for their thoughtfulness.

To develop critical appraisal skills, students must have time to reflect and think critically. Yet we have found in our personal experience that most of the alumni from our schools report having the opposite experience. Roth has written that most dental graduates felt their dental school experience was frustrating and that course content and assignments were either irrelevant or never put in context. ${ }^{22}$ Perhaps as problematic among alumni, according to Roth, is the feeling that the methods of clinical and preclinical instruction place a heavy emphasis on conformity. Yet, Roth argues, dental practice will be very different in the future from what it is today and dental education must be sensitive to a future of unprecedented discoveries. This is not to say that dental education should be set completely in an open schedule. What faculty should do, when appropriate, is lead the process and facilitate open exploration.

This raises the question of what we can do to foster successful problem-solving and innovative thinking. Berger recently wrote that there are several habits intrinsically linked to the ability of individuals to think strategically and function as successful innovators. ${ }^{23}$ The first of these habits is the ability 
to ask many questions. This activity is essential and needs to be fostered. The importance of asking "stupid questions" that challenge established truths may serve as the foundation for the design of new solutions. In the educational setting, the asking of fundamental "why?" questions encourages faculty members and students to reconsider established paradigms and reframe a solution that has not presented itself in the past.

The second value that provides a pathway for critical thinking is to focus on issues or circumstances that provoke a passionate response. This focus comes from addressing meaningful issues that inspire a leader's heart. For leaders to really be in a position to empathize, they must be willing to go where the group must go. The dedication required to observe people or the organization constitutes one of the best ways to identify unstated needs. "Focus groups and questionnaires don't cut it," says Berger. "Designers know that you must care enough to actually be present in people's lives." 23

The third concept that is essential for innovation and critical thinking is to take existing ideas or concepts and bring them together in novel ways. This strategy may represent a shortcut to innovation since new concepts do not need to be invented de novo. According to Pinsky et al., many leaders recognize that thinking laterally_ - "searching far and wide for ideas and influences" and "connecting ideas that might not seem to go together" - can represent a critical thinking strategy that can benefit from the experiences of other industries or influences. ${ }^{24}$

While original idea development is important, leaders must also be able to move design into action. Moving ideas from the chalkboard to prototype is not only a critical component of innovation but also provides opportunities to pilot models to provide feedback. This can be an iterative process that leads to improved models.

\section{Professionalism, Ethics, and Social Responsibility}

Ethical leadership is invaluable in any organization. The American Dental Association's Code of Ethics states that dental professionals should support patient autonomy and practice their profession with nonmaleficence, beneficence, justice, and veracity. ${ }^{25}$ The question is whether we as members of our profession want students to memorize these words or should we as educators provide support of real world scenarios that leaders and practitioners will face within their practice and professional framework?

Ethical leadership development must focus on content that extends beyond introspection within the profession. Pellegrino stated that the medical profession is a "moral community whose members are bound to each other by a set of commonly held ethical commitments and whose purpose is something more than self-interest. ${ }^{26}$ Haden et al. have written that academic dental institutions represent the introduction to this "moral community." relationship, academic institutions help to exhibit values that frame the dental profession's societal obligations. Academic dental institutions must prepare students to enter the dental profession as members of a community. Being a part of this community means participating in the organized profession.

Organized dentistry in turn can have a significant influence on state and federal policymakers, community leaders, and industry to help fulfill its moral duties. As part of this, the American Dental Association in its Code of Ethics and Professional Conduct states that "the dental profession should actively seek allies throughout the society on specific activities that will help improve access to care for all. ${ }^{25}$

Dentistry has a responsibility to develop the next generation of leaders for organized dentistry to continue the sense of social responsibility. Traditionally, the primary focus of dental school has been to get students ready for private practice. It should also include education as to the needs of the very young and the elderly, those with mental and physical disabilities, those who are medically compromised, and the underserved. A greater emphasis on delivering culturally competent care, public health issues, and the development of public health competencies should be openly discussed. Community-based programs should be a part of an interprofessional health system with physicians and nurses. ${ }^{27}$

Being able to effectively gain support for meaningful issues can make a significant difference in the success of the organization, including meeting the requirements of multiple regulatory agencies. Yet what are the obligations for dental leaders in the communities in which they serve? The development of skills focused on social responsibilities or advocacy represents a further area that future dental leaders should develop. We believe future dental leaders share an obligation to advocate for their patients and communities on issues pertaining to oral health 
(e.g., fluoride, sealants, and amalgam), but also to advocate for global issues pertaining to the health of their communities. In 2003, the American Dental Education Association (ADEA) report "Improving the Oral Health Status of All Americans: Roles and Responsibilities of Academic Dental Institutions" included the following in its list of recommendations for academic dental institutions in order to address the access problem: "Teaching and exhibiting values that prepare the student to enter the profession as a member of a moral community of oral health professionals with a commitment to the dental profession's societal obligations"; "Developing cultural competencies in their graduates and an appreciation for public health issues"; "Serving as effective providers, role models, and innovators in the delivery of oral health care to all populations"; and "Assisting in prevention, public health, and public education efforts to reduce health disparities in vulnerable populations." 27

Yet while dental schools are providing more experiences for students in the treatment of underserved communities and working interprofessionally, dental schools alone cannot solve many of the problems faced by the profession. For example, increasing student indebtedness has the potential to diminish the possibility that students will treat underserved populations once they graduate. The need to pay off student loans may be a deterrent to care providers' accepting reimbursement fees set by Medicaid or donating their time.

However, recognizing that these concerns represent ethical or moral issues provides a framework in which the profession must come to a solution. More importantly, it places courses in ethics into the real scenarios that students will need to address as professionals. Taking a position as a leader in the provision of care to people at risk and committing to service within the community provide opportunities for professionals to serve.

\section{Leadership Programs for Oral Health Students}

Building leadership skills and promoting collaboration will benefit the dental profession and the public that we serve. Leadership development provides an opportunity to learn from others and establish mentor-trainee relationships that may not be possible in the usual dental school setting. Ideally, this begins the first day in education, yet the dental school curriculum is often very dense. Moreover, to establish best practices for an effective leadership program for dental students is complicated by two facts: there are few models in dentistry on which to base an analysis, and, more importantly, the definition of what constitutes leadership in dentistry and what training is necessary to become a leader is complex. This is particularly true when one considers the many facets and competing concerns, ideas, and visions that leadership in our profession entails.

The most expedient path to establishing a leadership development program is to take existing ideas, concepts, or teaching elements and bring them together in novel ways. This strategy may represent a shortcut to innovation since new concepts do not need to be invented from scratch. Thinking laterally may represent a critical thinking strategy that can benefit from the experiences of others. ${ }^{24}$ Many of the existing dental leadership programs focused on preprofessional dentists have borrowed heavily from leadership programs designed with a focus on business leadership. ${ }^{28-30}$ These programs are attractive in that they carry with them an appeal to students who often find access to business professionals invigorating and relevant. For faculty members involved in program design, borrowing from business ventures is attractive not only to cover content but minimize the impact on faculty productivity. These programs have received significant support from the practicing community and clearly take advantage of resources available within a campus setting. ${ }^{28}$ How these programs are tailored for the oral health student and their needs is a challenge.

A second model, one developed and used at the University of Michigan, is to emphasize active learning while students work in groups or on individual leadership projects. ${ }^{31}$ Students learn negotiation skills, planning, interpersonal skills, and professionalism within a setting they choose and may to continue to participate in after graduation. The projects are left to the individual students or groups. This strategy emphasizes the need for passion for the project to be successful. This mode of learning is supplemented by invited speakers who address leadership within the realm of dentistry (e.g., practice management, education, research, public health, professional organizations) and from other professional settings.

There are several benefits to such a model. First, the program content can address the immediate 
professional needs and interests of the participants. Faculty members from across the campus can be incorporated into the program and may serve as a starting point for interprofessional collaboration. Thus, the program can serve as an opportunity to engage other professionals and stakeholders who do not usually participate in dental education. One of the major benefits of such a program is also the ability for dental students to network and participate in recruiting and hosting speakers.

The challenge with this design is that it can be labor-intensive from a faculty member's perspective. The best results are achieved when faculty members are engaged in active mentoring. An aspect of this program design that may make faculty members uncomfortable is the reality that they may not control the learning process. Thus, while much can be learned when positive results are achieved, faculty members must accept that in some instances failure is a valuable outcome and may result in an iterative process that leads to improved models.

A third model for leadership training of dental students is a blended model. The advantage of this model is that it can be less faculty-intensive while providing for opportunities for cross-campus and invited speakers to participate in the program. This model also provides greater flexibility for students who are interested in leadership development but for whom a leadership vendor may not appeal. This design benefits from students' working more independently on leadership projects while being less time-intensive for faculty mentors.

\section{Assessing Leadership Competence}

The assessment of reaching competence in leadership will undoubtedly be linked to the value of leadership programs in oral health in the educational setting. Yet objective measures of leadership competence are difficult to agree upon, for there are numerous examples of exceptional leaders who excel in one or several areas but not in others. Realistically, the achievement of competence in leadership will undoubtedly be determined through peer and self-assessments. In fact, this area of program design is exceptionally difficult to establish without the realization that what must be assessed is the journey rather than the destination. Nevertheless, the ADEA Leadership Institute surveyed its alumni to determine their perceptions of the benefits from the course. ${ }^{32}$ Not surprisingly, the respondents "reported that the most beneficial experiences included networking with the program participants, advisors, and instructors; self-discovery through self-assessments and evaluations; and a $360^{\circ}$ feedback process to provide additional reflection about areas for improvement." The results of this study on faculty development are consistent with those reported for leadership programs for dental and dental hygiene students. ${ }^{28-31}$

For example, feedback from the program at the University of Michigan suggests students feel that faculty members are more responsive to their needs, listen to them, and treat them as junior colleagues in the development of the program. In fact, the program has evolved into a venue for novel scholarship..$^{24,33,34}$ Moreover, such a program can serve as a vehicle for addressing faculty shortages ${ }^{35,36}$ by introducing students to academic careers. However, we urge administrators to take a long view of leadership programs since attaining leadership positions can take decades. Interim measures are often best assessed by asking the participants themselves if the program made a difference in their careers.

\section{Conclusions}

Leadership training for dental students represents a new opportunity that has largely gone unexplored in most dental schools. Many aspects of existing dental school curricula could be used to provide the basis of leadership training (e.g., practice management, diversity, research training). These programs can be faculty-intensive, yet by working on a leadership project, faculty members also may take new or additional paths in scholarship. Students' working in teams with faculty members to address a problem in research, policy, practice, or education encourages independent thought and project design to achieve leadership goals and provides a real-world setting for the development of critical thinking skills and lifelong learning.

We believe that a focus on leadership studies in the oral health professions is essential for the oral health of our nation so that professionals with the knowledge to best serve the public's needs will be prepared with both the skills and mindset to change the culture of our profession in a positive direction. We as educators must provide tangible examples and opportunities to all of our students to build within the profession the infrastructure to lead oral health 
policy. There are many paths and roles in leadership students can take. The most important point is that we must establish a dialogue within the education community so that when our graduates see and seek out opportunities to provide positive change, they will seize the opportunity - not just for the good of their practices or their own recognition, but to honor the oath they take when they enter the profession.

\section{Acknowledgments}

The authors would like to thank their parent institutions and deans for support during their participation in the American Dental Education Association (ADEA) Leadership Institute. The authors would like to acknowledge the help of Dr. Cecile Feldman, dean, University of Medicine \& Dentistry/New Jersey Dental School; Dr. Karl Haden, president, Academy for Academic Leadership, and his staff; and other fellows in the ADEA Leadership Institute. Dr. Taichman is supported by grants from the National Institutes of Health (DK082481, RC1DE020721, AR056893, CA093900) and the Department of Defense (PC073952). Sincere appreciation also goes out to ADEA and the Alpha Omega Foundation for supporting Dr. Taichman as a Leonard Abrams Scholar during his participation in the ADEA Leadership Institute. Finally, we would like to acknowledge the support and encouragement of our families and our students, for whom this work is intended.

\section{REFERENCES}

1. Curtis EK. Under oath: examining the role of the Hippocratic oath in dentistry. J Am Coll Dent 1998;65:33-5.

2. Valachovic RW. Audacious leadership. J Am Coll Dent 2004;71:50-2.

3. Slavkin H. Leadership in research: organizing genius. J Am Coll Dent 2004;71:33-8.

4. Collins-Nakai R. Leadership in medicine. McGill J Med 2006;9:68-73.

5. Walton JN, Clark DC, Glick N. An outcomes assessment of a hybrid-PBL course in treatment planning. J Dent Educ 1997;61(4):361-7.

6. Scannapieco FA. Formal debate: an active learning strategy. J Dent Educ 1997;61(12):955-61.

7. Rubin RW. Developing cultural competence and social responsibility in preclinical dental students. J Dent Educ 2004;68(4):460-7.

8. Masella RS. Confronting shibboleths of dental education. J Dent Educ 2005;69(10):1089-94.

9. DePaola DP. Leadership: creating a culture of caring. J Am Coll Dent 2004;71:45-9.

10. Bramson JB. Leadership: a new look, a new time, and established leadership. J Am Coll Dent 2004;71:39-44.
11. Philabosian D. Leadership as a passionate expression. J Am Coll Dent 2004;71:30-2.

12. Volpe A. Is there anything left for the next generation of leaders? J Am Coll Dent 2004;71:26-9.

13. Christensen GJ. Fourteen characteristics of effective leaders in dentistry. J Am Coll Dent 2004;71:18-21.

14. Slavkin H. Leadership for health care in the 21 st century: a personal perspective. J Healthcare Leadership 2010;2:35-41.

15. DePaola DP, Slavkin HC. Reforming dental health professions education: a white paper. J Dent Educ 2004;68(11):1139-50.

16. Kouzes J, Posner B. Academic administrator's guide to exemplary leadership. San Francisco: Jossey-Bass, 2003.

17. Lucas A. Strengthening departmental leadership: a teambuilding guide for chairs in colleges and universities. San Francisco: Jossey-Bass, 2004.

18. Bolman J, Deal TE. Reframing organizations: artistry, choice, and leadership. San Francisco: Jossey-Bass, 2008.

19. Finestquotes. At: www.finestquotes.com. Accessed: November 12, 2010.

20. Detsky AS. How to be a good academic leader. J Gen Intern Med 2011;26(1):88-90.

21. Kanter RM. Column: think outside the building. Harvard Business Review, March 2010. At: http://hbr.org/2010/03/ column-think-outside-the-building/ar/1. Accessed: November 20, 2010.

22. Roth K. Dental education: a leadership challenge for dental educators and practitioners. J Dent Educ 2007;71(8): 983-7.

23. Berger W. The four phases of design thinking. HBR Blog Network, July 29, 2010. At: http://blogs.hbr.org/ cs/2010/07/the four phases of design thin.html. Accessed: November 20, 2010.

24. Pinsky HM, Taichman RS, Sarment DP. Adaptation of airline crew resource management principles to dentistry. J Am Dent Assoc 2010;141:1010-8.

25. ADA principles of ethics and code of professional conduct. Chicago: American Dental Association, 2011.

26. Pellegrino ED. The medical profession as a moral community. Bull N Y Acad Med 1990;66:221-32.

27. Haden NK, Catalanotto FA, Alexander CJ, Bailit H, Battrell A, Broussard J Jr, et al. Improving the oral health status of all Americans: roles and responsibilities of academic dental institutions - the report of the ADEA president's commission. J Dent Educ 2003;67(5):563-83.

28. Slavkin HC, Lawrence LL. Incorporating leadership knowledge and skills into the dental education community. J Dent Educ 2007;71(6):708-12.

29. Victoroff KZ, Schneider K, Perry C. Tomorrow's leaders, starting today: a pilot leadership development program for dental students. J Dent Educ 2009;73(3):311-8.

30. Victoroff KZ, Schneider K, Perry C. Leadership development for dental students: what do students think? J Dent Educ 2008;72(4):982-8.

31. Taichman RS, Green TG, Polverini PJ. Creation of a scholars program in dental leadership (SPDL) for dental and dental hygiene students. J Dent Educ 2009;73(10): 1139-43. 
32. Haden NK, Ranney RR, Weinstein G, Breeding LC, Bresch JE, Valachovic RW. Leadership development in dental education: report on the ADEA Leadership Institute, 2000-08. J Dent Educ 2010;74(3):331-51.

33. Le TT, Scheller EL, Pinsky HM, Stefanac SJ, Taichman RS. Ability of dental students to deliver oxygen in a medical emergency. J Dent Educ 2009;73(4):499-508.

34. Stratz A, Taylor N. Can we build it? Yes we can! Access. July 2008.
35. Haden NK, Beemsterboer PL, Weaver RG, Valachovic RW. Dental school faculty shortages increase: an update on future dental school faculty. J Dent Educ 2000;64(9):657-73.

36. Nunn PJ, Gadbury-Amyot CC, Battrell A, Bruce SI, Hanlon LL, Kaiser C, Purifoy-Seldon B. The current status of allied dental faculty: a survey report. J Dent Educ 2004;68(3):329-44. 